\title{
Echogenicity of the Common Carotid Artery Intima-Media Complex in Stroke
}

\section{Authors:}

Kunihiko Aizawa, Salim Elyas, Damilola D Adingupu, Francesco Casanova, Kim M Gooding, Angela C Shore, W David Strain, Phillip E Gates

\section{Affiliation:}

Diabetes and Vascular Medicine Research Centre, University of Exeter Medical School, Barrack Road, Exeter, EX2 5AX, UK

NIHR Exeter Clinical Research Facility, Barrack Road, Exeter, EX2 5DW, UK

Royal Devon and Exeter NHS Foundation Trust, Barrack Road, EX2 5DW, Exeter, UK

\section{Running Headline:}

IMT echogenicity in stroke

\section{Corresponding Author:}

Kunihiko Aizawa, PhD

Diabetes and Vascular Medicine Research Centre

University of Exeter Medical School

Barrack Road, Exeter

EX2 5AX, UK

+44 1392403081 (TEL)

+44 1392403027 (FAX)

k.aizawa@exeter.ac.uk 


\section{ABSTRACT:}

Grey-scale median of the common carotid artery intima-media complex (IM-GSM) characterizes the arterial wall composition and low IM-GSM is associated with an increased cardiovascular mortality in the elderly. We aimed to determine differences in IM-GSM between a cohort with cerebrovascular disease and a healthy cohort. Eighty-two healthy individuals (CTRL: $63.2 \pm 8.7 y r s)$ and 96 patients with either stroke or transient ischemic

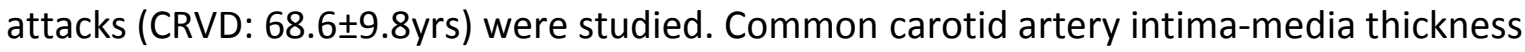
(cIMT) and IM-GSM obtained by ultrasound were analysed using semi-automated edgedetection software. IM-GSM was significantly lower in CRVD than CTRL $(106 \pm 30$ vs $124 \pm 27$ au, $p<0.001)$. IM-GSM was similar between the infarct and non-infarct sides in CRVD. In the pooled cohort of all participants, the lower the quartile of IM-GSM, the greater cIMT and carotid artery remodelling were. These results suggest the presence of an altered atherosclerotic phenotype in the intima-media complex of CVRD patients that can be detected by ultrasound.

\section{Key Words:}

Ultrasound, Stroke, Intima-media thickness, Echogenicity, Carotid artery 


\section{INTRODUCTION:}

Atherosclerosis is importantly involved in the aetio-pathology of a large proportion of strokes. Common carotid artery intima-media thickness (cIMT) by B-mode ultrasound is a widely used assessment of atherosclerotic vascular risk, is a predictor for stroke incidence (Bots et al. 1997), and is associated with prevalence of stroke (Ebrahim et al. 1999). However, a greater cIMT by itself does not necessarily indicate the presence of atherosclerosis or an atherosclerotic process occurring within the arterial wall. In fact, the age-related increase in cIMT may occur without overt atherosclerosis in humans (Spence 2006), and cIMT is mainly predicted by age and blood pressure in multiple regression analysis (Al-Shali et al. 2005). These issues illustrate a potential drawback of cIMT to evaluate atherosclerotic vascular changes and indicate a need for an assessment that better characterizes changes to the arterial wall.

Differences in the composition of the arterial wall and plaque have been shown to produce variations in the ultrasound signal returned from a vessel (Goncalves et al. 2004). Grey scale median of the common carotid artery intima-media complex (IM-GSM) is based on variations in echogenicity of cIMT that have been shown to characterize the composition of the arterial wall. Low IM-GSM also reflects more generalized atherosclerotic vulnerability (Lind et al. 2007) and the lowest tertile of IM-GSM was associated with an 8-fold increase in CV mortality compared with the mid-tertile in an elderly male population (Wohlin et al. 2009). Plaque echogenicity provides useful information about the vulnerability of plaques (e.g. stable or rupture-prone unstable plaque) (Mathiesen and Johnsen 2009) and is associated with increased risk of stroke (Polak et al. 1998; Gronholdt et al. 2001; Mathiesen et al. 2001) and cardiovascular (CV) events (Honda et al. 2004; Reiter et al. 2008). IM-GSM is 
closely related to carotid plaque echogenicity (Lind et al. 2007), suggesting that the biological mechanism underlying echogenicity of arterial wall may be similar between cIMT and plaque. Because it is not possible to obtain histological data from the common carotid artery of patients, an alternative method to characterise the appearance of the arterial wall may therefore be useful to monitor early atherosclerotic processes in the carotid artery before overt plaque develops.

IM-GSM may also provide a useful measurement for the evaluation of therapeutic interventions aimed at reducing vascular risk. Combining IM-GSM with established measurements, such as cIMT and vascular remodelling, has the potential to identify clustering of vascular biomarkers that allow early identification of pathology and improves patient stratification and management. It may also be useful in studying in vivo pathophysiology, and 'intermediate' biomarkers may be useful in evaluating interventions that target vascular disease. In this study, we set out to determine the utility of three ultrasound-based biomarkers of the common carotid artery: arterial wall echogenicity (IMGSM), arterial wall thickness (cIMT), and vascular remodelling (wall to lumen ratio; WLR). We took three approaches to this initial investigation of these biomarkers. First, we compared these biomarkers in an apparently healthy cohort with those in a cohort with recently diagnosed cerebrovascular disease (stroke or transient ischemic attacks; TIA). Wall thickness and vascular remodelling are known to vary from person to person, but the influence of those variations on IM-GSM is unknown. Second, we compared the biomarkers in the right and left carotid arteries within patients with cerebrovascular disease. This allowed us to compare arteries with identical 'traditional' risk factors. Previous studies have shown bilateral differences in cIMT in middle-aged and older non-lacunar cerebral infarct 
patients (Onbas et al. 2005), but it is unknown if bilateral differences in cIMT also reflect differences in arterial wall composition. As part of this analysis, we also compared vascular biomarkers from the affected-and non-affected sides of the brain in the cerebrovascular disease group. Finally, we explored the associations between IM-GSM, CIMT and WLR in a pooled data set.

\section{MATERIALS AND METHODS:}

In total, 178 individuals $(66.1 \pm 9.6 y r s, 72 F)$ participated in this study. Participants were allocated to the cerebrovascular disease group (CRVD: $n=96,68.6 \pm 9.8 y r s, 30 F)$ or the control group (CTRL: $n=82,63.2 \pm 8.7 y r s, 42 F)$. Individuals in CRVD had a stroke $(n=46)$ or TIA $(n=50)$ diagnosed within the three months before the first study visit, and were recruited from the daily stroke clinic and the acute stroke unit at the Royal Devon and Exeter Hospital, Exeter, UK. Individuals with cardioembolic, or haemorrhagic stroke/TIA were excluded. Individuals in the control group had no overt CV disease, cerebrovascular disease, type 2 diabetes, hypertension, or dyslipidaemia. In the morning of the study visit, participants were asked to abstain from taking any medications. UK National Research Ethics Service South West Committee approved all study procedures and written informed consent was obtained from all individuals.

\section{Experimental Procedures}

Participants attended the laboratory after an overnight fast and blood samples were drawn before they ate a standardized meal. After resting supine for $10 \mathrm{~min}$, blood pressure was measured using an automated oscillometric device (M5-I, Omron, Japan). 
The carotid arteries were assessed non-invasively using a Doppler ultrasound machine with a high-resolution linear array transducer (SSD-5500 SV, Aloka, Japan). Subjects lay supine on an examination bed with the head turned $\sim 45^{\circ}$ away from the examined side. The settings of the ultrasound machine were optimised, and the time-gain compensation was set in the neutral position. The B-mode common carotid artery images with satisfactory quality were stored in the ultrasound machine, and were downloaded later for off-line analysis. All images were taken at the R-wave of the ECG.

CIMT and IM-GSM were analyzed using semi-automated edge-detection software (Artery Measurement System: ver 2.02, Gothenburg, Sweden) (Liang et al. 2000). The software automatically identified a $10 \mathrm{~mm}$-long segment (from the beginning of the bulb) of the near wall-lumen boundary and the far wall IMT of the common carotid artery free from plaques (defined as $>50 \%$ increase in cIMT compared to adjacent cIMT). This automated analysis could be adjusted manually if the identification of borders was not appropriate. IMGSM was analysed using the same region of interest as cIMT. The software calculated IMGSM by analysing individual pixels within the region of interest on a scale from 0 (black) to 255 (white). For calibration, the lumen was used for the reference value for black and the brightest area of the adventitia was used for the reference value for white. cIMT was defined as the height between the lumen-intima and the media-adventitia borders at the far wall. WLR was defined as the ratio of cIMT to the lumen diameter. For all the carotid artery variables, data from the right and left sides of the common carotid arteries were averaged, and mean values were used for statistical analysis (except for the left and right side comparison, and the infarct- and non-infarct side comparison in CRVD). Using the software, intra-observer variability for repeated assessments (20 images, at least 1 week apart) of the 
carotid artery diameter, IMT and IM-GSM was assessed and the coefficient of variation was $1.3 \%$ for lumen diameter, $1.4 \%$ for cIMT, and $1.2 \%$ for IM-GSM, respectively.

\section{Statistical analysis}

All data are presented as means $\pm S D$ for variables with normal distribution, and median (interquartile range) for variables with skewed distribution. A chi-square test was used to examine the differences in categorical variables between CRVD and CTRL. Independent samples t-test for variables with normal distribution, and a Mann-Whitney U test for variables with skewed distribution were used to examine the differences in variables between CRVD and CTRL. We used a Kruskal-Wallis test to determine differences between the quartiles of IM-GSM that were derived from the pooled data set of all participants

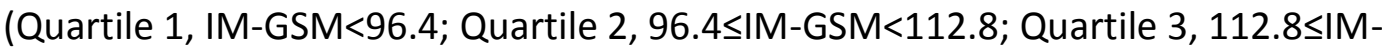

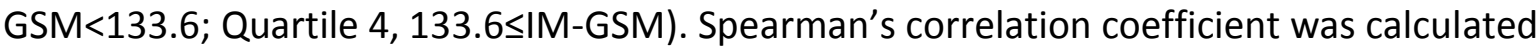
to examine the associations between the quartiles of IM-GSM and carotid artery variables. Significance was set at $p<0.05$. Statistical analysis was performed using IBM SPSS Statistics version 21 (IBM, Armonk, NY). A post-hoc power calculation revealed that we had 99\% power to detect a statistically significant difference in IM-GSM between CTRL and CRVD.

\section{RESULTS:}

A typical example of ultrasound images of the common carotid artery far-wall intima-media complex from a healthy individual (a) and stroke patient (b) is shown in Figure 1. 
Characteristics of CRVD and CTRL are shown in Table 1. The participants in CRVD were older than those in CTRL $(p<0.001)$. There were more male participants in CRVD than in CTRL ( $p=0.007)$. BMI of CRVD was greater than CTRL $(p<0.001)$. Total, LDL and HDL cholesterol concentrations were higher in CTRL than those of CRVD (all $p<0.001$ ). However, total and LDL cholesterol concentrations were not different between the groups after controlling for the statin treatment (data not shown). Lumen diameter, mean cIMT, and maximum cIMT were greater in CRVD than CTRL (all $p<0.001$ ) and IM-GSM was significantly lower in CRVD than CTRL $(p<0.001)$. These differences were independent of age and sex.

Carotid artery variables in CRVD divided into the right and left sides are shown in Table 2-1. The right side lumen diameter was greater than the left side $(p=0.003)$. Mean IMT, maximum IMT, WLR, and IM-GSM were all similar between the sides in this group. Carotid artery variables in CRVD divided into the infarct- and non-infarct sides are shown in Table 2-2. All the variables were similar between the infarct and non-infarct side in this group.

Table 3 shows the pooled data set of characteristics derived from all study participants, divided by the quartiles of IM-GSM. The participants in lower IM-GSM quartiles were older $(p=0.014)$. There was a significant increase in BMI with the reduction in IM-GSM $(p=0.004)$. Total $(p=0.006)$ and LDL cholesterol $(p=0.014)$ concentration was significantly greater with increased IM-GSM, but this was not the case after controlling for statin treatment (data not shown). Systolic blood pressure was significantly higher with the lower IM-GSM ( $p=0.036)$, but this association did not remain after controlling for anti- 
hypertensive treatment (data not shown). The number of individuals with a stroke/TIA, hypertension and dyslipidaemia was higher with the lower IM-GSM (all $p<0.001$ ).

Carotid artery variables derived from the pooled data set of all study participants divided by the quartiles of IM-GSM are shown in Table 4. There was a significant increase in mean cIMT, maximum cIMT and WLR with the reduction in IM-GSM (all $p<0.001$ ). These associations were independent of age, BMI, total and LDL cholesterol, systolic blood pressure, and medical history (stroke/TIA, hypertension and dyslipidaemia). There was also a significant inverse association between IM-GSM and mean $\operatorname{cIMT}(\rho=-0.508)$, maximum $\operatorname{cIMT}(\rho=-0.435)$ and WLR $(\rho=-0.445$, all $p<0.001$ : Figure 2$)$. Lumen diameter was similar among the quartiles.

\section{DISCUSSION:}

The salient findings of this study are as follows. First, IM-GSM was lower in CRVD compared with CTRL. Second, there was no bilateral difference in IM-GSM between the right and left sides or between the infarct and the non-infarct sides in CRVD. Third, lower IM-GSM was associated with greater cIMT and WLR in the pooled data set. These results show that IM-GSM is lower in the presence of stroke/TIA, suggesting the presence of an altered atherosclerotic phenotype in these patients. The results also suggest that this altered phenotype may be systemic in CRVD patients, rather than restricted to the infarct side. The inverse association observed between cIMT, WLR and IM-GSM indicates that these alterations in the arterial wall may be linked, raising a possibility of common mechanistic involvement and clustering of vascular risk factors. To our knowledge, this is the first study to show differences in IM-GSM between CRVD and healthy cohorts, demonstrating the 
potential for IM-GSM to provide additional and relevant information about the status of the arterial wall during a routine ultrasound examination.

\section{IM-GSM between the cerebrovascular disease and control groups}

IM-GSM was lower in CRVD than CTRL in this study, indicating a worse phenotype. The only comparable data are from sub-group analysis ( $2 \%$ of 942 participants) of an elderly cohort where IM-GSM of stroke sufferers was also lower than that of stroke-free subjects (Andersson et al. 2009). The majority of data derived from GSM has been from studies of carotid plaque. Echolucent plaque on the B-mode ultrasound image has been reported to be indicative of histological features of vulnerability, such as high lipid and macrophage content (Gronholdt 1999), intra-plaque haemorrhage (Gronholdt et al. 2002) and neovascularization (Coli et al. 2008). There is also an association between echolucent plaque and increased risk for ischemic stroke (Polak et al. 1998; Gronholdt et al. 2001; Mathiesen et al. 2001). Given the close relationship between plaque echogenicity and IM-GSM (Lind et al. 2007), it is plausible that reduced IM-GSM observed in CRVD could be explained by vascular changes similar to those of plaque. With an ability to make serial measurements, IM-GSM has the potential to monitor changes in the status of the arterial wall over time.

\section{IM-GSM between the right and left carotid arteries, and the infarct and non-infarct sides in the cerebrovascular disease group}

Onbas et al (2005) showed that cIMT on the infarct side was greater than that of the non-infarct side in non-lacunar cerebral infarct patients (Onbas et al. 2005). Rodriguez Hernandez et al (2003) have also shown that there was a relationship between cIMT and the infarct side in non-lacunar stroke patients in a population-based stroke registry. These 
findings imply an association between the infarct side of the brain and atherosclerotic vascular changes that could be detected by IM-GSM. However, in our dataset, IM-GSM was similar between the right and left sides, and also between the infarct and non-infarct sides in CRVD. This suggests that atherosclerotic vascular changes may progress systemically in CRVD. Our CIMT data also show that both mean and maximum cIMT were similar between the right and left sides, and between the infarct and non-infarct sides. A potential reason for this disparity between the previous studies (Rodriguez Hernandez et al. 2003; Onbas et al. $2005)$ and ours is that we included both lacunar $(n=42)$ and non-lacunar $(n=54)$ stroke/TIA patients. However, neither patient group in our study showed a significant difference in IMGSM between the infarct and non-infarct sides when analysed separately (lacunar, $104.9 \pm 27.7$ vs $109.5 \pm 34.7 \mathrm{au}$; non-lacunar, $103.7 \pm 23.1$ vs $105.3 \pm 28.5 \mathrm{au})$, in agreement with our principal finding.

\section{Associations between carotid artery structure and IM-GSM}

Although our study design does not allow us to establish a cause-and-effect relationship between wall thickness, vascular remodelling and IM-GSM, this is, to our knowledge, the first study to suggest that vascular remodelling modulates pre-clinical atherosclerosis in vivo. An inverse association between cIMT and IM-GSM has previously been reported in a population of 70 year-olds (Andersson et al. 2009) and in a middle-aged and older population with low CV risk but increased cIMT (Peters et al. 2012). Consistent with these findings, our data also showed an inverse association between cIMT and the quartiles of IM-GSM. We also showed an inverse association between WLR and the quartiles of IM-GSM, extending our understanding of the association between carotid artery structure and IM-GSM. Specifically, WLR, the lumen diameter-corrected wall thickness 
which serves as a marker of vascular remodelling (Schiffrin 2004), showed an inverse association with IM-GSM. This indicates that concentric remodelling (increased cIMT relative to lumen diameter) exhibits more atherosclerotic vascular changes and eccentric remodelling (reduced cIMT relative to lumen diameter) exhibits less atherosclerotic vascular change. Thus, we speculate that IM-GSM is influenced by both cIMT and remodelling that alters lumen diameter, such that arterial walls with similar cIMT will have different IM-GSM if lumen diameter is different.

\section{Clinical implications}

An advantage of IM-GSM is that it can be performed in individuals without overt plaque, and can evaluate vascular changes at different stages of the atherosclerotic CV disease continuum. This could extend the use of existing ultrasound assessment to identify individuals at risk of atherosclerosis early in the aetio-pathology of CV disease (Wohlin et al. 2009) and enable early management and intervention. In particular, this could be helpful in borderline risk individuals where the use of classical risk stratification tools, such as the Framingham risk scale, could potentially over- or underestimate CV disease risk.

Because we included a large number of patients and an older control group, we expected to find carotid plaque in a proportion of participants. At least one carotid artery plaque was found in $80 \%$ of CRVD and $50 \%$ of CTRL participants. Whereas IM-GSM is closely related to carotid plaque echogenicity (Lind et al. 2007), which is associated with increased risk of stroke (Polak et al. 1998; Gronholdt et al. 2001; Mathiesen et al. 2001), it is currently unknown whether IM-GSM predicts future stroke events independently of the presence of 
carotid plaque and/or the degree of echogenicity of carotid plaque. A longitudinal study will be necessary to answer this question.

Consistent with previous studies (Andersson et al. 2009; Peters et al. 2012), our IMGSM data correlated with BMI. This raises the possibility that IM-GSM might be amenable to beneficial modification (increased IM-GSM) by controlling BMI and related CV risk factors. A 3-year treatment with a $\beta$-blocker showed a significant increase in plaque echogenicity (Ostling et al. 2011). A post-hoc analysis of the METEOR study has also suggested a beneficial effect of 2-year rosuvastatin treatment on IM-GSM in individuals with low IMGSM at baseline (Lind et al. 2012). Additional studies are essential to establish the effect of CV risk factor modification on IM-GSM.

\section{Limitations}

First, although histological information of IM-GSM could be of benefit, it is not possible to obtain histological samples for IM-GSM from living persons without an indication for carotid endarterectomy. Nor is it possible to obtain serial histological samples to determine if changes in carotid IM-GSM are explained by compositional changes to the arterial wall. However, as plaque echogenicity reflects histological plaque composition (Goncalves et al. 2004), IM-GSM may also be determined by the properties of the arterial wall. IM-GSM also has some advantages over histology as serial measurements are possible that allow changes in IM-GSM over time to be monitored. Accumulating evidence suggests that characterisation of the ultrasound appearance of vessels may have clinical utility, although we acknowledge that additional insight from histological studies and virtual histology would be informative. Second, differences in gain settings between individuals 
could influence IM-GSM, but Peters et al (2013) report that variability in gain setting did not change the direction as well as magnitude of the relationship between echogenicity, CV risk factors and the observed rates of changes in echogenicity (Peters et al. 2013). We also use a calibration procedure to standardise IM-GSM analysis that has been shown to reduce variability in gain settings (Peters et al. 2013). Third, differences in IM-GSM between studies may be explained by the use of different machines, but our data were obtained using the same ultrasound machine for all participants. We acknowledge that there will be a need for standardization on the IM-GSM assessment before implementing it as a diagnostic parameter. This may include the development of software for IM-GSM that could be incorporated into commercial ultrasound systems, and of a phantom to calibrate IM-GSM Bmode images. Finally, an inherent limitation that applies to all ultrasound vascular assessment is the influence of the tissue composition between the ultrasound probe and target. This could have influenced our data because there was an inverse association between IM-GSM and BMI in this study. However, the observed associations between IMGSM and carotid artery biomarkers were independent of BMI. Thus, we think that IM-GSM scores obtained from the intima-media more closely reflect changes in the composition of the arterial wall, and not the tissue composition between the probe and the artery.

\section{CONCLUSIONS:}

IM-GSM was lower in stroke/TIA patients compared with control, but there was no bilateral difference in IM-GSM in these patients. We also found an inverse association between wall thickness, vascular remodelling and IM-GSM. These results suggest the presence of an altered atherosclerotic phenotype in stroke/TIA patients that may be systemic, rather than restricted to the infarct side. The inverse association between wall 
thickness, vascular remodelling and IM-GSM suggests that alterations in the composition of the carotid artery wall may be influenced by the extent of arterial remodelling, and that these vascular risk factors cluster in the carotid artery. IM-GSM may thus have a potential to provide additional and relevant information about the status of the arterial wall during a routine ultrasound examination in individuals with cerebrovascular disease.

\section{CONFLICTS OF INTEREST:}

Nothing to declare.

\section{AKNOWLEDGEMENT:}

This study was supported in part by the NIHR Exeter Clinical Research Facility. The views expressed are those of the authors and not necessarily those of the NHS, the NIHR, or the Department of Health. We would like to thank South West Stroke Research Network for their help in recruitment, and the staff of the Diabetes and Vascular Medicine Research Centre, NIHR Exeter Clinical Research Facility, University of Exeter Medical School, for their valuable assistance to carry out this study.

\section{REFERENCES:}

Al-Shali K, House AA, Hanley AJ, Khan HM, Harris SB, Mamakeesick M, Zinman B, Fenster A, Spence JD, Hegele RA. Differences between carotid wall morphological phenotypes measured by ultrasound in one, two and three dimensions. Atherosclerosis 2005;178:319-25.

Andersson J, Sundstrom J, Gustavsson T, Hulthe J, Elmgren A, Zilmer K, Zilmer M, Lind L. Echogenecity of the carotid intima-media complex is related to cardiovascular risk factors, 
dyslipidemia, oxidative stress and inflammation: the Prospective Investigation of the Vasculature in Uppsala Seniors (PIVUS) study. Atherosclerosis 2009;204:612-8.

Bots ML, Hoes AW, Koudstaal PJ, Hofman A, Grobbee DE. Common carotid intima-media thickness and risk of stroke and myocardial infarction: the Rotterdam Study. Circulation 1997;96:14327.

Coli S, Magnoni M, Sangiorgi G, Marrocco-Trischitta MM, Melisurgo G, Mauriello A, Spagnoli L, Chiesa R, Cianflone D, Maseri A. Contrast-enhanced ultrasound imaging of intraplaque neovascularization in carotid arteries: correlation with histology and plaque echogenicity. J Am Coll Cardiol 2008;52:223-30.

Ebrahim S, Papacosta O, Whincup P, Wannamethee G, Walker M, Nicolaides AN, Dhanjil S, Griffin M, Belcaro G, Rumley A, Lowe GD. Carotid plaque, intima media thickness, cardiovascular risk factors, and prevalent cardiovascular disease in men and women: the British Regional Heart Study. Stroke 1999;30:841-50.

Goncalves I, Lindholm MW, Pedro LM, Dias N, Fernandes e Fernandes J, Fredrikson GN, Nilsson J, Moses J, Ares MP. Elastin and calcium rather than collagen or lipid content are associated with echogenicity of human carotid plaques. Stroke 2004;35:2795-800.

Gronholdt ML. Ultrasound and lipoproteins as predictors of lipid-rich, rupture-prone plaques in the carotid artery. Arterioscler Thromb Vasc Biol 1999;19:2-13.

Gronholdt ML, Nordestgaard BG, Bentzon J, Wiebe BM, Zhou J, Falk E, Sillesen H. Macrophages are associated with lipid-rich carotid artery plaques, echolucency on B-mode imaging, and elevated plasma lipid levels. J Vasc Surg 2002;35:137-45.

Gronholdt ML, Nordestgaard BG, Schroeder TV, Vorstrup S, Sillesen H. Ultrasonic echolucent carotid plaques predict future strokes. Circulation 2001;104:68-73.

Honda O, Sugiyama S, Kugiyama K, Fukushima H, Nakamura S, Koide S, Kojima S, Hirai N, Kawano H, Soejima H, Sakamoto T, Yoshimura M, Ogawa H. Echolucent carotid plaques predict future coronary events in patients with coronary artery disease. J Am Coll Cardiol 2004;43:1177-84. 
Liang Q, Wendelhag I, Wikstrand J, Gustavsson T. A multiscale dynamic programming procedure for boundary detection in ultrasonic artery images. IEEE Trans Med Imaging 2000;19:127-42.

Lind L, Andersson J, Ronn M, Gustavsson T. The echogenecity of the intima-media complex in the common carotid artery is closely related to the echogenecity in plaques. Atherosclerosis 2007;195:411-4.

Lind L, Peters SA, den Ruijter HM, Palmer MK, Grobbee DE, Crouse JR, 3rd, O'Leary DH, Evans GW, Raichlen JS, Bots ML, Group MS. Effect of rosuvastatin on the echolucency of the common carotid intima-media in low-risk individuals: the METEOR trial. J Am Soc Echocardiogr 2012;25:1120-7.

Mathiesen EB, Bonaa $\mathrm{KH}$, Joakimsen $\mathrm{O}$. Echolucent plaques are associated with high risk of ischemic cerebrovascular events in carotid stenosis: the tromso study. Circulation 2001;103:2171-5. Mathiesen EB, Johnsen SH. Ultrasonographic measurements of subclinical carotid atherosclerosis in prediction of ischemic stroke. Acta Neurol Scand Suppl 2009;189:68-72.

Onbas O, Kantarci M, Okur A, Bayraktutan U, Edis A, Ceviz N. Carotid intima-media thickness: is it correlated with stroke side? Acta Neurol Scand 2005;111:169-71.

Ostling G, Goncalves I, Wikstrand J, Berglund G, Nilsson J, Hedblad B. Long-term treatment with lowdose metoprolol $\mathrm{CR} / \mathrm{XL}$ is associated with increased plaque echogenicity: the Beta-blocker Cholesterol-lowering Asymptomatic Plaque Study (BCAPS). Atherosclerosis 2011;215:440-5.

Peters SA, Bots ML, Lind L, Groenewegen KA, de Korte C, den Ruijter HM, group Ms. The impact of variability in ultrasound settings on the measured echolucency of the carotid intima-media. J Hypertens 2013;31:1861-7.

Peters SA, Lind L, Palmer MK, Grobbee DE, Crouse JR, 3rd, O'Leary DH, Evans GW, Raichlen J, Bots ML, den Ruijter HM, group Ms. Increased age, high body mass index and low HDL-C levels are related to an echolucent carotid intima-media: the METEOR study. J Intern Med 2012;272:257-66. 
Polak JF, Shemanski L, O'Leary DH, Lefkowitz D, Price TR, Savage PJ, Brant WE, Reid C. Hypoechoic plaque at US of the carotid artery: an independent risk factor for incident stroke in adults aged 65 years or older. Cardiovascular Health Study. Radiology 1998;208:649-54.

Reiter M, Effenberger I, Sabeti S, Mlekusch W, Schlager O, Dick P, Puchner S, Amighi J, Bucek RA, Minar E, Schillinger M. Increasing carotid plaque echolucency is predictive of cardiovascular events in high-risk patients. Radiology 2008;248:1050-5.

Rodriguez Hernandez SA, Kroon AA, van Boxtel MP, Mess WH, Lodder J, Jolles J, de Leeuw PW. Is there a side predilection for cerebrovascular disease? Hypertension 2003;42:56-60.

Schiffrin EL. Remodeling of resistance arteries in essential hypertension and effects of antihypertensive treatment. Am J Hypertens 2004;17:1192-200.

Spence JD. Technology Insight: ultrasound measurement of carotid plaque--patient management, genetic research, and therapy evaluation. Nature clinical practice Neurology 2006;2:611-9.

Wohlin M, Sundstrom J, Andren B, Larsson A, Lind L. An echolucent carotid artery intima-media complex is a new and independent predictor of mortality in an elderly male cohort. Atherosclerosis 2009;205:486-91.

\section{FIGURE CAPTIONS LIST:}

Figure 1. Example of ultrasound images of the carotid artery far-wall intima-media complex from a healthy individual (a) and stroke patient (b). A similar ultrasound gain setting was used for both acquisitions. Darker cIMT produces lower scores and is associated with increased vascular risk. cIMT was similar between individuals $(0.928 \mathrm{~mm}$ and $0.939 \mathrm{~mm})$ but IM-GSM was lower in the stroke patients (91 au) than the healthy participants (144 au).

Figure 2. Carotid artery lumen diameter (top left), mean intima-media thickness (top right), maximum intima-media thickness (bottom left) and wall-to-lumen ratio (bottom right) data 
divided by the quartiles of IM-GSM in the pooled data set of all study participants. Data are median (95\% confidence interval). 
Table 1. Selected characteristics, medical history and carotid artery biomarkers of the study participants.

\begin{tabular}{|c|c|c|c|}
\hline & Control & $\begin{array}{c}\text { Cerebrovascular } \\
\text { Disease }\end{array}$ & $p$ \\
\hline Age, yrs & $63.2 \pm 8.7$ & $68.6 \pm 9.8$ & $<0.001$ \\
\hline Sex, M/F & $40 / 42$ & $66 / 30$ & 0.007 \\
\hline Smoking, $\mathrm{n}$ & 4 & 7 & 0.505 \\
\hline $\mathrm{BMI}, \mathrm{kg} / \mathrm{m}^{2}$ & $25.5 \pm 3.6$ & $28.0 \pm 4.0$ & $<0.001$ \\
\hline Total $\mathrm{CHOL}, \mathrm{mmol} / \mathrm{l}$ & $5.70(5.00-6.30)$ & $3.90(3.38-4.60)$ & $<0.001$ \\
\hline LDL CHOL, mmol/l & $3.35(2.88-3.91)$ & $1.82(1.49-2.44)$ & $<0.001$ \\
\hline $\mathrm{HDL} \mathrm{CHOL}, \mathrm{mmol} / \mathrm{l}$ & $1.68(1.34-2.00)$ & $1.35(1.11-1.71)$ & $<0.001$ \\
\hline Triglycerides, mmol/l & $1.03(0.83-1.49)$ & $1.05(0.83-1.50)$ & 0.897 \\
\hline Glucose, $\mathrm{mmol} / \mathrm{l}$ & $5.00(4.80-5.50)$ & $5.20(4.73-5.68)$ & 0.256 \\
\hline Systolic BP, mmHg & $138.5 \pm 18.8$ & $141.9 \pm 16.8$ & 0.207 \\
\hline Diastolic BP, mmHg & $79.2 \pm 9.0$ & $78.9 \pm 8.6$ & 0.820 \\
\hline Type 2 Diabetes, $\mathrm{n}$ & 0 & 10 & 0.003 \\
\hline Hypertension, $\mathrm{n}$ & 0 & 70 & $<0.001$ \\
\hline$C A D, n$ & 0 & 12 & 0.001 \\
\hline$P A D, n$ & 0 & 1 & 0.354 \\
\hline Dyslipidaemia, n & 0 & 89 & $<0.001$ \\
\hline Lumen diameter, $\mathrm{mm}$ & $5.68 \pm 0.61$ & $6.08 \pm 0.66$ & $<0.001$ \\
\hline cIMT mean, $\mathrm{mm}$ & $0.76(0.69-0.87)$ & $0.87(0.80-0.95)$ & $<0.001$ \\
\hline cIMT maximum, $\mathrm{mm}$ & $0.90(0.78-1.03)$ & $1.02(0.93-1.17)$ & $<0.001$ \\
\hline WLR, au & $0.137(0.120-0.156)$ & $0.143(0.129-0.163)$ & 0.087 \\
\hline Carotid plaque, $\mathrm{n}$ & 41 & 77 & $<0.001$ \\
\hline IM-GSM, au & $123.6 \pm 27.4$ & $105.8 \pm 24.3$ & $<0.001$ \\
\hline
\end{tabular}

Data are shown as means $\pm \mathrm{SD}$ or median (interquartile range). $\mathrm{BMI}$, body mass index; $\mathrm{CHOL}$, cholesterol; LDL, low-density lipoprotein; HDL, high-density lipoprotein; BP, blood pressure; CAD, coronary artery disease; PAD, peripheral artery disease; CIMT, common carotid artery intima-media thickness; WLR, wall-to-lumen ratio; Carotid plaque, number of individuals with the presence of at least one carotid plaque; IM-GSM, grey scale median of the carotid artery intima-media complex. 
Table 2-1. Carotid artery biomarkers of the right and left sides in CRVD.

\begin{tabular}{lccc}
\hline & Right side & Left side & $p$ \\
\hline Lumen diameter, mm & $6.11 \pm 0.70$ & $5.97 \pm 0.66$ & 0.003 \\
IMT mean, mm & $0.89 \pm 0.17$ & $0.88 \pm 0.17$ & 0.875 \\
IMT maximum, mm & $1.05 \pm 0.21$ & $1.05 \pm 0.19$ & 0.807 \\
WLR, au & $0.147 \pm 0.032$ & $0.149 \pm 0.029$ & 0.408 \\
IM-GSM, au & $106.2 \pm 29.4$ & $105.1 \pm 27.4$ & 0.722 \\
\hline
\end{tabular}

Table 2-2. Carotid artery biomarkers of the infarct- and non-infarct sides in CVRD.

\begin{tabular}{lccc}
\hline & Infarct side & Non-infarct side & $p$ \\
\hline Lumen diameter, mm & $6.01 \pm 0.72$ & $6.07 \pm 0.65$ & 0.214 \\
IMT mean, mm & $0.88 \pm 0.16$ & $0.89 \pm 0.18$ & 0.246 \\
IMT maximum, mm & $1.04 \pm 0.19$ & $1.05 \pm 0.21$ & 0.467 \\
WLR, au & $0.147 \pm 0.028$ & $0.149 \pm 0.033$ & 0.524 \\
IM-GSM, au & $105.1 \pm 27.9$ & $106.2 \pm 28.8$ & 0.708 \\
\hline
\end{tabular}

Data are shown as means \pm SD. Abbreviations as Table 1. 
Table 3. Selected characteristics and medical history of the all participants derived from the pooled data set divided by the quartiles of IMGSM.

\begin{tabular}{|c|c|c|c|c|c|}
\hline & Quartile1 (<96.4) & $\begin{array}{c}\text { Quartile2 (96.4- } \\
112.7)\end{array}$ & Quartile3 (112.8-133.5) & Quartile4 ( $\geq 133.6$ ) & $P$ \\
\hline Age, yrs & $68.5(63.3-76.3)$ & $65.0(61.5-74.0)$ & $63.0(55.5-68.5)$ & $65.0(60.0-69.0)$ & 0.014 \\
\hline Sex, M/F & $28 / 16$ & $30 / 15$ & $24 / 21$ & $24 / 20$ & 0.488 \\
\hline Smoking, $\mathrm{n}$ & 1 & 4 & 4 & 2 & 0.475 \\
\hline $\mathrm{BMI}, \mathrm{kg} / \mathrm{m}^{2}$ & $28.0(26.1-31.0)$ & $26.5(24.0-30.1)$ & $25.4(23.6-28.0)$ & $25.4(22.9-29.2)$ & 0.004 \\
\hline HDL CHOL, mmol/l & $1.50(1.13-1.83)$ & $1.37(1.18-1.80)$ & $1.54(1.28-1.92)$ & $1.56(1.21-1.94)$ & 0.493 \\
\hline Triglycerides, mmol/l & $1.26(0.94-1.57)$ & $1.06(0.67-1.41)$ & $1.07(0.85-1.53)$ & $0.95(0.84-1.27)$ & 0.169 \\
\hline Glucose, $\mathrm{mmol} / \mathrm{l}$ & $5.40(4.80-5.80)$ & $5.20(4.80-5.55)$ & $5.00(4.70-5.55)$ & $5.00(4.70-5.50)$ & 0.178 \\
\hline Systolic BP, mmHg & $142.8(134.0-159.0)$ & 141.9 (131.9-148.9) & $133.3(122.5-143.3)$ & $140.7(126.7-153.6)$ & 0.036 \\
\hline Hypertension, $n$ & 28 & 22 & 10 & 10 & $<0.001$ \\
\hline CAD, $n$ & 3 & 5 & 3 & 1 & 0.429 \\
\hline PAD, $n$ & 0 & 1 & 0 & 0 & 0.396 \\
\hline Dyslipidaemia, $n$ & 29 & 29 & 18 & 13 & 0.001 \\
\hline IM-GSM, au & $79.8(72.9-91.6)$ & 102.5 (98.5-109.3) & $121.0(117-129.5)$ & $148.6(140.0-158.3)$ & $<0.001$ \\
\hline
\end{tabular}

Data are shown as median (interquartile range). Abbreviations as Table 1. 
Table 4. Carotid artery biomarkers of all participants derived from the pooled data set divided by the quartiles of IM-GSM.

\begin{tabular}{lccccc}
\hline & Quartile1 $(<96.4)$ & $\begin{array}{c}\text { Quartile2 }(96.4- \\
112.7)\end{array}$ & Quartile3 (112.8-133.5) & Quartile4 ( $\geq 133.6)$ & $P$ \\
\hline Lumen diameter, mm & $5.92(5.56-6.24)$ & $6.01(5.61-6.50)$ & $5.73(5.21-6.23)$ & $5.84(5.44-6.20)$ & 0.153 \\
IMT mean, mm & $0.93(0.84-1.05)$ & $0.87(0.78-0.99)$ & $0.75(0.69-0.86)$ & $0.74(0.64-0.85)$ & $<0.001$ \\
IMT maximum, mm & $1.06(0.98-1.20)$ & $1.01(0.92-1.17)$ & $0.90(0.80-1.04)$ & $0.88(0.76-1.02)$ & $<0.001$ \\
WLR, au & $0.161(0.141-0.180)$ & $0.146(0.125-0.164)$ & $0.134(0.122-0.146)$ & $0.128(0.112-0.143)$ & $<0.001$ \\
\hline
\end{tabular}

Data are shown as median (interquartile range). Abbreviations as Table 1. 
Figure 1.
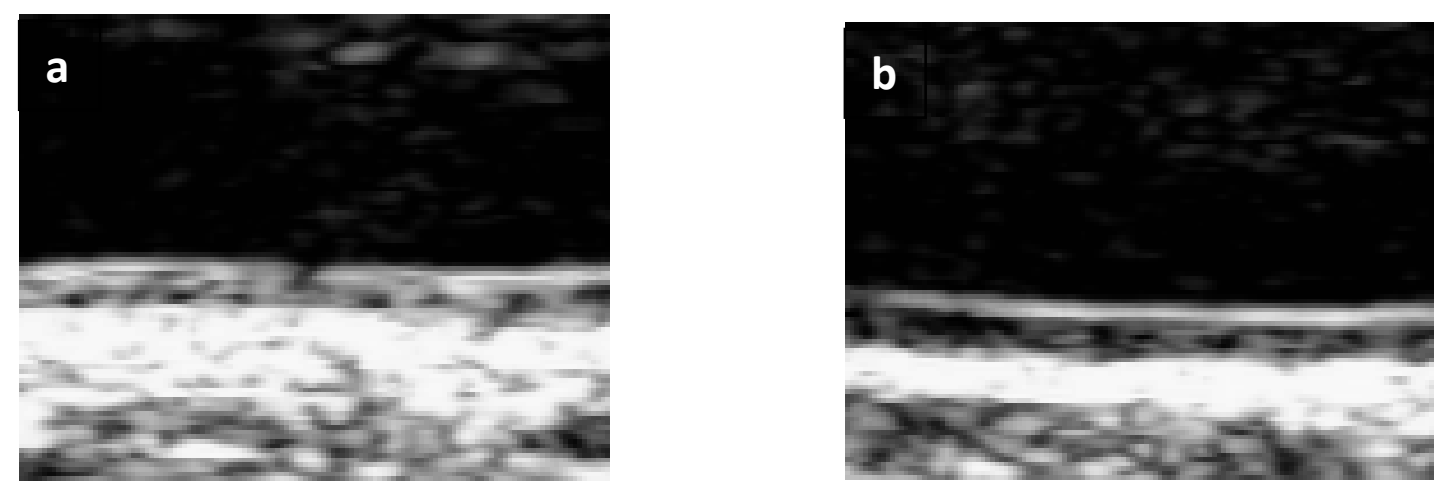
Figure 2.
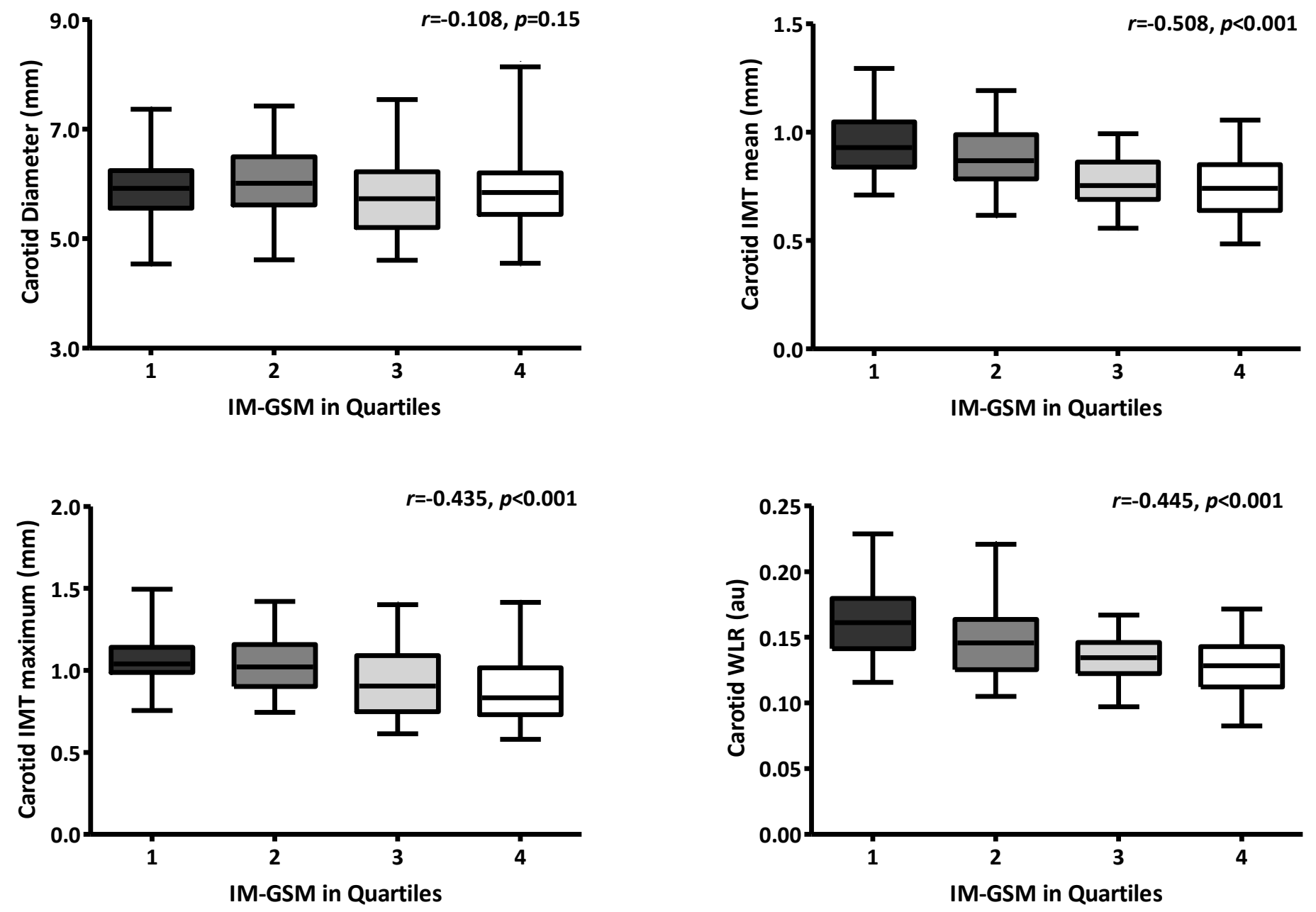